\title{
Availability and Profit Optimization of Series-Parallel System with Linear Consecutive Cold Standby Units
}

\author{
Muhammad Sagir Aliyu ${ }^{*}$, Ibrahim Yusuf' ${ }^{2}$ U. A. Ali ${ }^{1}$ \\ ${ }^{1}$ Department of Mathematics, Usmanu Dan Fodio University, Sokoto, Nigeria \\ ${ }^{2}$ Department of Mathematical Sciences, Bayero University, Kano, Nigeria \\ Email: ${ }^{*}$ muhammadsagiraliyu@yahoo.com, iyusuf.mth@buk.edu.ng, ubahamad@yahoo.co.uk
}

Received 16 January 2015; accepted 6 February 2015; published 10 February 2015

Copyright (C) 2015 by authors and Scientific Research Publishing Inc.

This work is licensed under the Creative Commons Attribution International License (CC BY). http://creativecommons.org/licenses/by/4.0/

\section{(c) (i) Open Access}

\begin{abstract}
In this paper, we study availability and profit optimization of a series-parallel system consisting of three subsystems $A, B$ and $C$ in which $A$ and $B$ are cold standby. Subsystem $A$ consists of linear consecutive $k$-out-of- $n$ units while subsystems $B$ and $C$ consist of a single unit each. The system works if any of $A$ or $B$ and $C$ work. The objective of this study is to maximize the steady-state availability and profit. To solve the optimization problem, different numbers of units for $n=2,3,4,5$ in subsystem $A$ are considered. Explicit expressions for busy period of repairmen, steady-state availability and profit function are derived using linear first order differential equations. Several cases are analyzed graphically for $n=2,3,4,5$ to investigate the effects of various system parameters on availability and profit. The paper also presents graphical comparison for specific values of system parameters and finds that the optimal system configuration is when $n=5$.
\end{abstract}

\section{Keywords}

Availability, Profit, Cold Standby, Optimization

\section{Introduction}

The series-parallel systems consist of subsystems connected in series where each subsystem consists of units arranged in parallel. Failure of any one of the subsystems leads to the failure of the system. These systems are used in industries, power stations, manufacturing, production and telecommunications. Due to their importance in promoting and sustaining industries and economy, reliability measures of such systems have become an area of interest. Among the reliability measures of interest there are the steady-state availability, busy period, profit

\footnotetext{
"Corresponding author.
}

How to cite this paper: Aliyu, M.S., Yusuf, I. and Ali, U.A. (2015) Availability and Profit Optimization of Series-Parallel System with Linear Consecutive Cold Standby Units. Applied Mathematics, 6, 332-344.

http://dx.doi.org/10.4236/am.2015.62032 
function and mean time to system failure (MTSF). Availability and profit of redundant systems can be enhanced using highly reliable structural system design. Improving the reliability and availability of system/subsystem leads to an increase in production and associated profit. Researches carried out on optimization problem for seriesparallel/ $k$-out-of- $n G$ systems can be found in Hu et al. [1], Khatab et al. [2] who analyzed the availability of $k$-out-of- $n G$ system with non identical components subject to repair priorities, Krishnan et al. [3] analyzed the reliability and profit analysis of repairable $k$-out-of- $n$ system with sensor, Juang et al. [4], Levitin [5], Li et al. [6] and Wang et al. [7]. Wang et al. [8] performed comparative analysis of availability among three systems with general repair times, reboot delay and switching failure. Wang et al. [9] performed comparative analysis of availability between two systems with warm standby units and different imperfect coverage. The problem considered in the present paper is different from the work of the above mentioned authors in the sense that a number of units incorporated in subsystem $A$ as a linear consecutive $k$-out-of- $n$. The contribution of this paper is twofold. First is to develop the explicit expressions for steady-state availability, busy period of repair man and profit function. Second is to perform numerical investigation on the effect of system parameters on reliability indices mentioned above. Models developed in this paper are found to be highly beneficial to engineers, maintenance managers, system designers and plant management for proper maintenance analysis, decision, and evaluation of performance. Comparisons are performed for $n=2,3,4,5$, for steady-state availability and profit based on assumed numerical values given to the system parameters.

The organization of the paper is as follows. Assumptions' of the study and states of the systems are presented in Section 2. Models formulations are given in Section 3. The results of our numerical simulations and discussions are presented in Section 4. Finally, we make a concluding remark in Section 5.

\section{Assumptions and States of the Systems}

\subsection{Assumptions}

1) The system is attended by three repairmen;

2) The failure and repair time are to be assumed exponential;

3) Units in subsystem $A$ are linear consecutive $k$-out-of- $n$;

4) Subsystem $A$ and $B$ are in cold standby;

5) Repair is instantaneous;

6) Repaired unit is as good as new.

\subsection{States of the System}

System I

$$
\begin{aligned}
& S_{0}\left(A_{1 O}, A_{2 S}, B_{S}, C_{O}\right), \\
& S_{1}\left(A_{1 R}, A_{2 O}, B_{S}, C_{O}\right), \\
& S_{2}\left(A_{1 W}, A_{2 R}, B_{O}, C_{O}\right), \\
& S_{3}\left(A_{1 G}, A_{2 S}, B_{S}, C_{R}\right), \\
& S_{4}\left(A_{1 W}, A_{2 G}, B_{S}, C_{R}\right), \\
& S_{5}\left(A_{1 W}, A_{2 R}, B_{W}, C_{G}\right), \\
& S_{6}\left(A_{1 W}, A_{2 R}, B_{G}, C_{R}\right) .
\end{aligned}
$$

System II

$$
\begin{aligned}
& S_{0}\left(A_{1 O}, A_{2 O}, A_{3 S}, B_{S}, C_{O}\right), \\
& S_{1}\left(A_{1 R}, A_{2 O}, A_{3 O}, B_{S}, C_{O}\right), \\
& S_{2}\left(A_{1 G}, A_{2 R}, A_{3 G}, B_{O}, C_{O}\right),
\end{aligned}
$$




$$
\begin{aligned}
& S_{3}\left(A_{1 W}, A_{2 R}, A_{3 G}, B_{O}, C_{O}\right), \\
& S_{4}\left(A_{1 G}, A_{2 G}, A_{3 S}, B_{S}, C_{R}\right), \\
& S_{5}\left(A_{1 R}, A_{2 G}, A_{3 G}, B_{S}, C_{R}\right), \\
& S_{6}\left(A_{1 G}, A_{2 R}, A_{3 G}, B_{R}, C_{G}\right), \\
& S_{7}\left(A_{1 G}, A_{2 R}, A_{3 G}, B_{G}, C_{R}\right), \\
& S_{8}\left(A_{1 W}, A_{2 R}, A_{3 G}, B_{R}, C_{G}\right), \\
& S_{9}\left(A_{1 W}, A_{2 R}, A_{3 G}, B_{G}, C_{R}\right) .
\end{aligned}
$$

System III

$$
\begin{aligned}
& S_{0}\left(A_{1 O}, A_{2 O}, A_{3 S}, A_{4 S}, B_{S}, C_{O}\right), \\
& S_{1}\left(A_{1 R}, A_{2 O}, A_{3 O}, A_{4 S}, B_{S}, C_{O}\right), \\
& S_{2}\left(A_{1 G}, A_{2 R}, A_{3 O}, A_{4 O}, B_{S}, C_{O}\right), \\
& S_{3}\left(A_{1 W}, A_{2 R}, A_{3 O}, A_{4 O}, B_{S}, C_{O}\right), \\
& S_{4}\left(A_{1 W}, A_{2 W}, A_{3 R}, A_{4 G}, B_{O}, C_{O}\right), \\
& S_{5}\left(A_{1 G}, A_{2 W}, A_{3 R}, A_{4 G}, B_{O}, C_{O}\right), \\
& S_{6}\left(A_{1 R}, A_{2 G}, A_{3 G}, A_{4 S}, B_{S}, C_{R}\right), \\
& S_{7}\left(A_{1 W}, A_{2 R}, A_{3 G}, A_{4 G}, B_{S}, C_{R}\right), \\
& S_{8}\left(A_{1 W}, A_{2 W}, A_{3 R}, A_{4 G}, B_{R}, C_{G}\right), \\
& S_{9}\left(A_{1 W}, A_{2 W}, A_{3 R}, A_{4 G}, B_{G}, C_{R}\right), \\
& S_{10}\left(A_{1 G}, A_{2 W}, A_{3 R}, A_{4 G}, B_{R}, C_{G}\right), \\
& S_{11}\left(A_{1 G}, A_{2 W}, A_{3 R}, A_{4 G}, B_{G}, C_{R}\right), \\
& S_{12}\left(A_{1 G}, A_{2 G}, A_{3 S}, A_{4 S}, B_{S}, C_{R}\right), \\
& S_{13}\left(A_{1 G}, A_{2 R}, A_{3 G}, A_{4 G}, B_{S}, C_{R}\right) .
\end{aligned}
$$

System IV

$$
\begin{aligned}
& S_{0}\left(A_{1 O}, A_{2 O}, A_{3 S}, A_{4 S}, A_{5 S}, B_{S}, C_{O}\right), \\
& S_{1}\left(A_{1 R}, A_{2 O}, A_{3 O}, A_{4 S}, A_{5 S}, B_{S}, C_{O}\right), \\
& S_{2}\left(A_{1 G}, A_{2 R}, A_{3 O}, A_{4 O}, A_{5 S}, B_{S}, C_{O}\right), \\
& S_{3}\left(A_{1 W}, A_{2 R}, A_{3 O}, A_{4 O}, A_{5 S}, B_{S}, C_{O}\right), \\
& S_{4}\left(A_{1 W}, A_{2 W}, A_{3 R}, A_{4 O}, A_{5 O}, B_{S}, C_{O}\right), \\
& S_{5}\left(A_{1 W}, A_{2 W}, A_{3 W}, A_{4 R}, A_{5 S}, B_{O}, C_{O}\right), \\
& S_{6}\left(A_{1 G}, A_{2 W}, A_{3 R}, A_{4 O}, A_{5 O}, B_{S}, C_{O}\right), \\
& S_{7}\left(A_{1 G}, A_{2 W}, A_{3 W}, A_{4 R}, A_{5 G}, B_{O}, C_{O}\right),
\end{aligned}
$$




$$
\begin{aligned}
& S_{8}\left(A_{1 G}, A_{2 G}, A_{3 G}, A_{4 S}, A_{5}, B_{S}, C_{R}\right), \\
& S_{9}\left(A_{1 R}, A_{2 G}, A_{3 G}, A_{4 S}, A_{5 S}, B_{S}, C_{R}\right), \\
& S_{10}\left(A_{1 W}, A_{2 R}, A_{3 G}, A_{4 S}, A_{5 S}, B_{S}, C_{R}\right), \\
& S_{11}\left(A_{1 W}, A_{2 W}, A_{3 R}, A_{4 G}, A_{5 S}, B_{G}, C_{R}\right), \\
& S_{12}\left(A_{1 W}, A_{2 W}, A_{3 W}, A_{4 R}, A_{5 G}, B_{R}, C_{G}\right), \\
& S_{13}\left(A_{1 W}, A_{2 W}, A_{3 W}, A_{4 R}, A_{5 G}, B_{G}, C_{R}\right), \\
& S_{14}\left(A_{1 G}, A_{2 R}, A_{3 G}, A_{4 G}, A_{5 S}, B_{S}, C_{R}\right), \\
& S_{15}\left(A_{1 G}, A_{2 W}, A_{3 R}, A_{4 G}, A_{5 G}, B_{S}, C_{R}\right), \\
& S_{16}\left(A_{1 G}, A_{2 W}, A_{3 W}, A_{4 R}, A_{5 G}, B_{R}, C_{R}\right), \\
& S_{17}\left(A_{1 G}, A_{2 W}, A_{3 W}, A_{4 R}, A_{5 G}, B_{G}, C_{R}\right) .
\end{aligned}
$$

\section{Models Formulation}

\subsection{Availability, Busy Period and Profit Modeling for $\boldsymbol{n}=2$}

Let $P(0)=\left[P_{0}(0), P_{1}(0), P_{2}(0), P_{3}(0), \cdots, P_{6}(0)\right]$ be the probability vector for system at time $t \geq 0$. Relating the state of the system at time $t$ and $t+\mathrm{d} t$, the differential equations for the system when $n=2$ can be expressed in the form:

$$
\frac{\mathrm{d}}{\mathrm{d} t}(P(t))=A_{1} P(t)
$$

where

$$
A_{1}=\left[\begin{array}{ccccccc}
-y_{11} & \alpha_{11} & 0 & \alpha_{2} & 0 & 0 & 0 \\
\beta_{11} & -y_{22} & \alpha_{12} & 0 & \alpha_{2} & 0 & 0 \\
0 & \beta_{12} & -y_{33} & 0 & 0 & \alpha_{1} & \alpha_{2} \\
\beta_{2} & 0 & 0 & -\alpha_{2} & 0 & 0 & 0 \\
0 & \beta_{2} & 0 & 0 & -\alpha_{2} & 0 & 0 \\
0 & 0 & \beta_{1} & 0 & 0 & -\alpha_{1} & 0 \\
0 & 0 & \beta_{2} & 0 & 0 & 0 & -\alpha_{2}
\end{array}\right]
$$

For the analysis of availability and busy period cases of system, we use the following procedure to obtain the steady-state availability, busy period and profit function. In steady-state, the derivatives of the state probabilities become zero and we obtain

$$
\left[\begin{array}{ccccccc}
-y_{11} & \alpha_{11} & 0 & \alpha_{2} & 0 & 0 & 0 \\
\beta_{11} & -y_{22} & \alpha_{12} & 0 & \alpha_{2} & 0 & 0 \\
0 & \beta_{12} & -y_{33} & 0 & 0 & \alpha_{1} & \alpha_{2} \\
\beta_{2} & 0 & 0 & -\alpha_{2} & 0 & 0 & 0 \\
0 & 0 & 0 & 0 & -\alpha_{2} & 0 & 0 \\
0 & 0 & \beta_{1} & 0 & 0 & -\alpha_{1} & 0 \\
0 & \beta_{2} & \beta_{2} & 0 & 0 & 0 & -\alpha_{2}
\end{array}\right]\left[\begin{array}{l}
P_{0}(t) \\
P_{1}(t) \\
P_{2}(t) \\
P_{3}(t) \\
P_{4}(t) \\
P_{5}(t) \\
P_{6}(t)
\end{array}\right]=\left[\begin{array}{l}
0 \\
0 \\
0 \\
0 \\
0 \\
0 \\
0
\end{array}\right]
$$

Replacing the last row of (2) with the normalizing condition below

$$
\sum_{i=0}^{6} P_{i}(\infty)=1
$$


to obtain the states probabilities $P_{0}(\infty), P_{1}(\infty), \cdots, P_{6}(\infty)$.

Let $T$ be the time to failure of the system for system.

The explicit expression for the steady-state availability is as follows:

The steady-state availability is given by

$$
A_{T 1}=P_{0}(\infty)+P_{1}(\infty)+P_{2}(\infty)=\frac{H_{1}}{D_{1}}
$$

From state 1 to 6 the repairmen are busy in those states repairing the failed units. Let $B_{T 1}(\infty)$ be the probabilities that the repairmen are busy in the states repairing the failed units. Using (2) and (3) above, the explicit expressions for the steady-state busy period of repairmen are as follows:

$$
B_{T 1}=P_{1}(\infty)+P_{2}(\infty)+P_{3}(\infty)+P_{4}(\infty)+P_{5}(\infty)+P_{6}(\infty)=\frac{H_{2}}{D_{1}}
$$

The system/subsystems/units are subjected to corrective maintenance at failure as can be observed in states 1 , 2, 3, 4, 5 and 6 of system I. In those states, the repairmen are busy performing corrective maintenance action to the system/subsystems/units at failure. The expected profit $P F_{1}$ per unit time incurred to the system in the steady-state is given by:

Profit $=$ total revenue generated - accumulated cost incurred due corrective maintenance to the failed system/ subsystems/units. Thus

$$
P F_{1}=C_{0} A_{T 1}(\infty)-C_{1} B_{T 1}(\infty)
$$

\subsection{Availability, Busy Period and Profit Modeling for $n=3$}

Let $P(0)=\left[P_{1}(0), P_{2}(0), P_{3}(0), \cdots, P_{9}(0)\right]$ be the probability vector for system at time $t \geq 0$. Relating the state of the system at time $t$ and $t+\mathrm{d} t$ the differential equations for the system when $n=3$ can be expressed in the form:

$$
A_{2}=\left[\begin{array}{cccccccccc}
-y_{1} & \alpha_{11} & \alpha_{12} & 0 & \alpha_{2} & 0 & 0 & 0 & 0 & 0 \\
\beta_{11} & -y_{2} & 0 & \alpha_{12} & 0 & \alpha_{2} & 0 & 0 & 0 & 0 \\
\beta_{12} & 0 & -y_{3} & 0 & 0 & 0 & \alpha_{1} & \alpha_{2} & 0 & 0 \\
0 & \beta_{12} & 0 & -y_{4} & 0 & 0 & 0 & 0 & \alpha_{1} & \alpha_{2} \\
\beta_{2} & 0 & 0 & 0 & -\alpha_{2} & 0 & 0 & 0 & 0 & 0 \\
0 & \beta_{2} & 0 & 0 & 0 & -\alpha_{2} & 0 & 0 & 0 & 0 \\
0 & 0 & \beta_{1} & 0 & 0 & 0 & -\alpha_{1} & 0 & 0 & 0 \\
0 & 0 & \beta_{2} & 0 & 0 & 0 & 0 & -\alpha_{2} & 0 & 0 \\
0 & 0 & 0 & \beta_{1} & 0 & 0 & 0 & 0 & -\alpha_{1} & 0 \\
0 & 0 & 0 & \beta_{2} & 0 & 0 & 0 & 0 & 0 & -\alpha_{2}
\end{array}\right]
$$

For the analysis of availability and busy period cases of system, we use the following procedure to obtain the 
steady-state availability, busy period and profit function. In steady-state, the derivatives of the state probabilities become zero and we obtain

$$
\left[\begin{array}{cccccccccc}
-y_{1} & \alpha_{11} & \alpha_{12} & 0 & \alpha_{2} & 0 & 0 & 0 & 0 & 0 \\
\beta_{11} & -y_{2} & 0 & \alpha_{12} & 0 & \alpha_{2} & 0 & 0 & 0 & 0 \\
\beta_{12} & 0 & -y_{3} & 0 & 0 & 0 & \alpha_{1} & \alpha_{2} & 0 & 0 \\
0 & \beta_{12} & 0 & -y_{4} & 0 & 0 & 0 & 0 & \alpha_{1} & \alpha_{2} \\
\beta_{2} & 0 & 0 & 0 & -\alpha_{2} & 0 & 0 & 0 & 0 & 0 \\
0 & \beta_{2} & 0 & 0 & 0 & -\alpha_{2} & 0 & 0 & 0 & 0 \\
0 & 0 & \beta_{1} & 0 & 0 & 0 & -\alpha_{1} & 0 & 0 & 0 \\
0 & 0 & \beta_{2} & 0 & 0 & 0 & 0 & -\alpha_{2} & 0 & 0 \\
0 & 0 & 0 & \beta_{1} & 0 & 0 & 0 & 0 & -\alpha_{1} & 0 \\
0 & 0 & 0 & \beta_{2} & 0 & 0 & 0 & 0 & 0 & -\alpha_{2}
\end{array}\right]\left[\begin{array}{l}
P_{0}(t) \\
P_{1}(t) \\
P_{2}(t) \\
P_{3}(t) \\
P_{4}(t) \\
P_{5}(t) \\
P_{6}(t) \\
P_{7}(t) \\
P_{8}(t) \\
P_{9}(t)
\end{array}\right]=\left[\begin{array}{l}
0 \\
0 \\
0 \\
0 \\
0 \\
0 \\
0 \\
0 \\
0 \\
0
\end{array}\right]
$$

Solving (8) and using the following normalizing condition

$$
\sum_{i=0}^{9} P_{i}(\infty)=1
$$

to obtain $P_{0}(\infty), P_{1}(\infty), P_{2}(\infty), \cdots, P_{9}(\infty)$.

The explicit expression for the steady-state availability is as follows:

$$
A_{T 2}(\infty)=P_{0}(\infty)+P_{1}(\infty)+P_{2}(\infty)+P_{3}(\infty)=\frac{H_{3}}{D_{2}}
$$

where

$$
\begin{gathered}
H_{3}=\alpha_{1} \alpha_{2} \alpha_{11} \alpha_{12}+\alpha_{1} \alpha_{2} \alpha_{12} \beta_{11}+\alpha_{1} \alpha_{2} \alpha_{11} \beta_{12}+\alpha_{1} \alpha_{2} \beta_{11} \beta_{12} \\
D_{2}=\alpha_{1} \alpha_{2} \alpha_{12} \beta_{11}+\alpha_{1} \alpha_{2} \beta_{11} \beta_{12}+\alpha_{1} \alpha_{2} \alpha_{11} \alpha_{12}+\alpha_{1} \alpha_{2} \alpha_{11} \beta_{12}+\alpha_{1} \alpha_{12} \beta_{2} \beta_{11} \\
+\alpha_{1} \beta_{2} \beta_{11} \beta_{12}+\alpha_{1} \alpha_{11} \alpha_{12} \beta_{2}+\alpha_{1} \alpha_{11} \beta_{2} \beta_{12}+\alpha_{2} \beta_{1} \beta_{11} \beta_{12}+\alpha_{1} \alpha_{11} \beta_{1} \beta_{12} .
\end{gathered}
$$

From state 1 to 9 the repairmen are busy in those states repairing the failed units. Let $B_{T 2}(\infty)$ be the probabilities that the repairmen are busy in those states repairing the failed units. Using (8) and (9) above, the explicit expressions for the steady-state busy period of repairmen are as follows:

$$
B_{T 2}(\infty)=P_{1}(\infty)+P_{2}(\infty)+P_{3}(\infty)+\cdots+P_{9}(\infty)=\frac{H_{4}}{D_{2}}
$$

The expected profit $P F_{2}$ per unit time incurred to the system in the steady-state is given by:

Profit $=$ total revenue generated - accumulated cost incurred due corrective maintenance to the failed system/ subsystems/units.

$$
P F_{2}=C_{0} A_{T 2}(\infty)-C_{1} B_{T 2}(\infty)
$$

\subsection{Availability, Busy Period and Profit Modeling for $n=4$}

Let $P(0)=\left[P_{0}(0), P_{1}(0), P_{2}(0), P_{3}(0), \cdots, P_{13}(0)\right]$ be the probability vector for system at time $t \geq 0$. Relating the state of the system at time $t$ and $t+\mathrm{d} t$ the differential equations for the system when $n=4$ can be expressed in the form:

$$
\frac{\mathrm{d}}{\mathrm{d} t}(P(t))=A_{3} P(t)
$$

where 


$$
\left.A_{3}=\begin{array}{cccccccccccccc}
-h_{1} & \alpha_{11} & \alpha_{12} & 0 & 0 & 0 & 0 & 0 & 0 & 0 & 0 & 0 & \alpha_{2} & 0 \\
\beta_{11} & -h_{2} & 0 & \alpha_{12} & 0 & 0 & \alpha_{2} & 0 & 0 & 0 & 0 & 0 & 0 & 0 \\
\beta_{12} & 0 & -h_{3} & 0 & 0 & \alpha_{13} & 0 & 0 & 0 & 0 & 0 & 0 & 0 & \alpha_{2} \\
0 & \beta_{12} & 0 & -h_{4} & \alpha_{13} & 0 & 0 & \alpha_{2} & 0 & 0 & 0 & 0 & 0 & 0 \\
0 & 0 & 0 & \beta_{13} & -h_{5} & 0 & 0 & 0 & \alpha_{1} & \alpha_{2} & 0 & 0 & 0 & 0 \\
0 & 0 & \beta_{13} & 0 & 0 & -h_{6} & 0 & 0 & 0 & 0 & \alpha_{1} & \alpha_{2} & 0 & 0 \\
0 & \beta_{2} & 0 & 0 & 0 & 0 & -\alpha_{2} & 0 & 0 & 0 & 0 & 0 & 0 & 0 \\
0 & 0 & 0 & \beta_{2} & 0 & 0 & 0 & -\alpha_{2} & 0 & 0 & 0 & 0 & 0 & 0 \\
0 & 0 & 0 & 0 & \beta_{1} & 0 & 0 & 0 & -\alpha_{1} & 0 & 0 & 0 & 0 & 0 \\
0 & 0 & 0 & 0 & \beta_{2} & 0 & 0 & 0 & 0 & -\alpha_{2} & 0 & 0 & 0 & 0 \\
0 & 0 & 0 & 0 & 0 & \beta_{1} & 0 & 0 & 0 & 0 & -\alpha_{1} & 0 & 0 & 0 \\
0 & 0 & 0 & 0 & 0 & \beta_{2} & 0 & 0 & 0 & 0 & 0 & -\alpha_{2} & 0 & 0 \\
\beta_{2} & 0 & 0 & 0 & 0 & 0 & 0 & 0 & 0 & 0 & 0 & 0 & -\alpha_{2} & 0 \\
0 & 0 & \beta_{2} & 0 & 0 & 0 & 0 & 0 & 0 & 0 & 0 & 0 & 0 & -\alpha_{2}
\end{array}\right]
$$

In steady-state, the derivatives of the state probabilities become zero and we obtain

$\left[\begin{array}{cccccccccccccc}-h_{1} & \alpha_{11} & \alpha_{12} & 0 & 0 & 0 & 0 & 0 & 0 & 0 & 0 & 0 & \alpha_{2} & 0 \\ \beta_{11} & -h_{2} & 0 & \alpha_{12} & 0 & 0 & \alpha_{2} & 0 & 0 & 0 & 0 & 0 & 0 & 0 \\ \beta_{12} & 0 & -h_{3} & 0 & 0 & \alpha_{13} & 0 & 0 & 0 & 0 & 0 & 0 & 0 & \alpha_{2} \\ 0 & \beta_{12} & 0 & -h_{4} & \alpha_{13} & 0 & 0 & \alpha_{2} & 0 & 0 & 0 & 0 & 0 & 0 \\ 0 & 0 & 0 & \beta_{13} & -h_{5} & 0 & 0 & 0 & \alpha_{1} & \alpha_{2} & 0 & 0 & 0 & 0 \\ 0 & 0 & \beta_{13} & 0 & 0 & -h_{6} & 0 & 0 & 0 & 0 & \alpha_{1} & \alpha_{2} & 0 & 0 \\ 0 & \beta_{2} & 0 & 0 & 0 & 0 & -\alpha_{2} & 0 & 0 & 0 & 0 & 0 & 0 & 0 \\ 0 & 0 & 0 & \beta_{2} & 0 & 0 & 0 & -\alpha_{2} & 0 & 0 & 0 & 0 & 0 & 0 \\ 0 & 0 & 0 & 0 & \beta_{1} & 0 & 0 & 0 & -\alpha_{1} & 0 & 0 & 0 & 0 & 0 \\ 0 & 0 & 0 & 0 & \beta_{2} & 0 & 0 & 0 & 0 & -\alpha_{2} & 0 & 0 & 0 & 0 \\ 0 & 0 & 0 & 0 & 0 & \beta_{1} & 0 & 0 & 0 & 0 & -\alpha_{1} & 0 & 0 & 0 \\ 0 & 0 & 0 & 0 & 0 & \beta_{2} & 0 & 0 & 0 & 0 & 0 & -\alpha_{2} & 0 & 0 \\ \beta_{2} & 0 & 0 & 0 & 0 & 0 & 0 & 0 & 0 & 0 & 0 & 0 & -\alpha_{2} & 0 \\ 0 & 0 & \beta_{2} & 0 & 0 & 0 & 0 & 0 & 0 & 0 & 0 & 0 & 0 & -\alpha_{2}\end{array}\right]\left[\begin{array}{l}P_{0}(t) \\ P_{1}(t) \\ P_{2}(t) \\ P_{3}(t) \\ P_{4}(t) \\ P_{5}(t) \\ P_{6}(t) \\ P_{7}(t) \\ P_{8}(t) \\ P_{9}(t) \\ P_{10}(t) \\ P_{11}(t) \\ P_{12}(t) \\ P_{13}(t)\end{array}\right]=\left[\begin{array}{l}0 \\ 0 \\ 0 \\ 0 \\ 0 \\ 0 \\ 0 \\ 0 \\ 0 \\ 0 \\ 0 \\ 0 \\ 0\end{array}\right]$

Solving (8) and using the following normalizing condition

$$
\sum_{i=0}^{13} P_{i}(\infty)=1
$$

and obtain $P_{0}(\infty), P_{1}(\infty), P_{2}(\infty), \cdots, P_{12}(\infty), P_{13}(\infty)$. 
The explicit expression for the steady-state availability is as

$$
A_{\text {T3 }}(\infty)=P_{0}(\infty)+P_{1}(\infty)+P_{2}(\infty)+P_{3}(\infty)+P_{4}(\infty)+P_{5}(\infty)=\frac{H_{5}}{D_{3}}
$$

From state 1 to 13 the repairmen are busy in those states repairing the failed units. Let $B_{T 3}(\infty)$ be the probabilities that the repairmen are busy in those states repairing the failed units. Using (14) and (15) above, the explicit expressions for the steady-state busy period of repairmen are as follows:

$$
B_{\text {Тз }}(\infty)=P_{1}(\infty)+P_{2}(\infty)+P_{3}(\infty)+P_{4}(\infty)+\cdots+P_{13}(\infty)=\frac{H_{6}}{D_{3}}
$$

The expected profit $\mathrm{PF}_{3}$ per unit time incurred to the system in the steady-state is given by:

Profit = total revenue generated - accumulated cost incurred due corrective maintenance to the failed system/ subsystems/units.

$$
P F_{3}=C_{0} A_{T 3}(\infty)-C_{1} B_{T 3}(\infty)
$$

\subsection{Availability, Busy Period and Profit Modeling for $\boldsymbol{n}=\mathbf{5}$}

Let $P(0)=\left[P_{1}(0), P_{2}(0), P_{3}(0), \cdots, P_{17}(0)\right]$ be the probability vector for system at time $t \geq 0$. Relating the state of the system at time $t$ and $t+\mathrm{d} t$ the differential equations for $n=5$ can be expressed in the form:

$$
\frac{\mathrm{d}}{\mathrm{d} t}(P(t))=A_{4} P(t)
$$

where

$$
A_{4}=\left[\begin{array}{cccccccccccccccccc}
-k_{1} & \alpha_{11} & \alpha_{12} & 0 & 0 & 0 & 0 & 0 & \alpha_{2} & 0 & 0 & 0 & 0 & 0 & 0 & 0 & 0 & 0 \\
\beta_{11} & -k_{2} & 0 & \alpha_{12} & 0 & 0 & 0 & 0 & 0 & \alpha_{2} & 0 & 0 & 0 & 0 & 0 & 0 & 0 & 0 \\
\beta_{12} & 0 & -k_{3} & 0 & 0 & 0 & \alpha_{13} & 0 & 0 & 0 & 0 & 0 & 0 & 0 & \alpha_{2} & 0 & 0 & 0 \\
0 & \beta_{12} & 0 & -k_{4} & \alpha_{13} & 0 & 0 & 0 & 0 & 0 & \alpha_{2} & 0 & 0 & 0 & 0 & 0 & 0 & 0 \\
0 & 0 & 0 & \beta_{13} & -k_{5} & \alpha_{14} & 0 & 0 & 0 & 0 & 0 & \alpha_{2} & 0 & 0 & 0 & 0 & 0 & 0 \\
0 & 0 & 0 & 0 & \beta_{14} & -k_{6} & 0 & 0 & 0 & 0 & 0 & 0 & \alpha_{1} & \alpha_{2} & 0 & 0 & 0 & 0 \\
0 & 0 & \beta_{13} & 0 & 0 & 0 & -k_{7} & \alpha_{14} & 0 & 0 & 0 & 0 & 0 & 0 & 0 & \alpha_{2} & 0 & 0 \\
0 & 0 & 0 & 0 & 0 & 0 & \beta_{14} & -k_{8} & 0 & 0 & 0 & 0 & 0 & 0 & 0 & 0 & \alpha_{1} & \alpha_{2} \\
\beta_{2} & 0 & 0 & 0 & 0 & 0 & 0 & 0 & -\alpha_{2} & 0 & 0 & 0 & 0 & 0 & 0 & 0 & 0 & 0 \\
0 & \beta_{2} & 0 & 0 & 0 & 0 & 0 & 0 & 0 & -\alpha_{2} & 0 & 0 & 0 & 0 & 0 & 0 & 0 & 0 \\
0 & 0 & 0 & \beta_{2} & 0 & 0 & 0 & 0 & 0 & 0 & -\alpha_{2} & 0 & 0 & 0 & 0 & 0 & 0 & 0 \\
0 & 0 & 0 & 0 & \beta_{2} & 0 & 0 & 0 & 0 & 0 & 0 & -\alpha_{2} & 0 & 0 & 0 & 0 & 0 & 0 \\
0 & 0 & 0 & 0 & 0 & \beta_{1} & 0 & 0 & 0 & 0 & 0 & 0 & -\alpha_{1} & 0 & 0 & 0 & 0 & 0 \\
0 & 0 & 0 & 0 & 0 & \beta_{2} & 0 & 0 & 0 & 0 & 0 & 0 & 0 & -\alpha_{2} & 0 & 0 & 0 & 0 \\
0 & 0 & \beta_{2} & 0 & 0 & 0 & 0 & 0 & 0 & 0 & 0 & 0 & 0 & 0 & -\alpha_{2} & 0 & 0 & 0 \\
0 & 0 & 0 & 0 & 0 & 0 & \beta_{2} & 0 & 0 & 0 & 0 & 0 & 0 & 0 & 0 & -\alpha_{2} & 0 & 0 \\
0 & 0 & 0 & 0 & 0 & 0 & 0 & \beta_{1} & 0 & 0 & 0 & 0 & 0 & 0 & 0 & 0 & -\alpha_{1} & 0 \\
0 & 0 & 0 & 0 & 0 & 0 & 0 & \beta_{2} & 0 & 0 & 0 & 0 & 0 & 0 & 0 & 0 & 0 & -\alpha_{2}
\end{array}\right]
$$




$$
\begin{aligned}
& k_{4}=\left(\alpha_{12}+\beta_{13}+\beta_{2}\right), \\
& k_{5}=\left(\alpha_{13}+\beta_{14}+\beta_{2}\right), \\
& k_{6}=\left(\alpha_{14}+\beta_{1}+\beta_{2}\right), \\
& k_{7}=\left(\alpha_{13}+\beta_{14}+\beta_{2}\right), \\
& k_{8}=\left(\alpha_{14}+\beta_{1}+\beta_{2}\right) .
\end{aligned}
$$

In steady-state, the derivatives of the state probabilities become zero and we obtain

$$
\left[\begin{array}{cccccccccccccccccc}
-k_{1} & \alpha_{11} & \alpha_{12} & 0 & 0 & 0 & 0 & 0 & \alpha_{2} & 0 & 0 & 0 & 0 & 0 & 0 & 0 & 0 & 0 \\
\beta_{11} & -k_{2} & 0 & \alpha_{12} & 0 & 0 & 0 & 0 & 0 & \alpha_{2} & 0 & 0 & 0 & 0 & 0 & 0 & 0 & 0 \\
\beta_{12} & 0 & -k_{3} & 0 & 0 & 0 & \alpha_{13} & 0 & 0 & 0 & 0 & 0 & 0 & 0 & \alpha_{2} & 0 & 0 & 0 \\
0 & \beta_{12} & 0 & -k_{4} & \alpha_{13} & 0 & 0 & 0 & 0 & 0 & \alpha_{2} & 0 & 0 & 0 & 0 & 0 & 0 & 0 \\
0 & 0 & 0 & \beta_{13} & -k_{5} & \alpha_{14} & 0 & 0 & 0 & 0 & 0 & \alpha_{2} & 0 & 0 & 0 & 0 & 0 & 0 \\
0 & 0 & 0 & 0 & \beta_{14} & -k_{6} & 0 & 0 & 0 & 0 & 0 & 0 & \alpha_{1} & \alpha_{2} & 0 & 0 & 0 & 0 \\
0 & 0 & \beta_{13} & 0 & 0 & 0 & -k_{7} & \alpha_{14} & 0 & 0 & 0 & 0 & 0 & 0 & 0 & \alpha_{2} & 0 & 0 \\
0 & 0 & 0 & 0 & 0 & 0 & \beta_{14} & -k_{8} & 0 & 0 & 0 & 0 & 0 & 0 & 0 & 0 & \alpha_{1} & \alpha_{2} \\
\beta_{2} & 0 & 0 & 0 & 0 & 0 & 0 & 0 & -\alpha_{2} & 0 & 0 & 0 & 0 & 0 & 0 & 0 & 0 & 0 \\
0 & \beta_{2} & 0 & 0 & 0 & 0 & 0 & 0 & 0 & -\alpha_{2} & 0 & 0 & 0 & 0 & 0 & 0 & 0 & 0 \\
0 & 0 & 0 & \beta_{2} & 0 & 0 & 0 & 0 & 0 & 0 & -\alpha_{2} & 0 & 0 & 0 & 0 & 0 & 0 & 0 \\
0 & 0 & 0 & 0 & \beta_{2} & 0 & 0 & 0 & 0 & 0 & 0 & -\alpha_{2} & 0 & 0 & 0 & 0 & 0 & 0 \\
0 & 0 & 0 & 0 & 0 & \beta_{1} & 0 & 0 & 0 & 0 & 0 & 0 & -\alpha_{1} & 0 & 0 & 0 & 0 & 0 \\
0 & 0 & 0 & 0 & 0 & \beta_{2} & 0 & 0 & 0 & 0 & 0 & 0 & 0 & -\alpha_{2} & 0 & 0 & 0 & 0 \\
0 & 0 & \beta_{2} & 0 & 0 & 0 & 0 & 0 & 0 & 0 & 0 & 0 & 0 & 0 & -\alpha_{2} & 0 & 0 & 0 \\
0 & 0 & 0 & 0 & 0 & 0 & \beta_{2} & 0 & 0 & 0 & 0 & 0 & 0 & 0 & 0 & -\alpha_{2} & 0 & 0 \\
0 & 0 & 0 & 0 & 0 & 0 & 0 & \beta_{1} & 0 & 0 & 0 & 0 & 0 & 0 & 0 & 0 & -\alpha_{1} & 0 \\
0 & 0 & 0 & 0 & 0 & 0 & 0 & \beta_{2} & 0 & 0 & 0 & 0 & 0 & 0 & 0 & 0 & 0 & -\alpha_{2}
\end{array}\right]\left[\begin{array}{c}
P_{0}(t) \\
P_{1}(t) \\
P_{2}(t) \\
P_{3}(t) \\
P_{4}(t) \\
P_{5}(t) \\
P_{6}(t) \\
P_{7}(t) \\
P_{8}(t) \\
P_{9}(t) \\
P_{10}(t) \\
P_{11}(t) \\
P_{12}(t) \\
P_{13}(t) \\
P_{14}(t) \\
P_{15}(t) \\
P_{16}(t) \\
P_{17}(t)
\end{array}\right]=\left[\begin{array}{c}
0 \\
0 \\
0 \\
0 \\
0 \\
0 \\
0 \\
0 \\
0 \\
0 \\
0 \\
0 \\
0 \\
0 \\
0 \\
0 \\
0
\end{array}\right.
$$

to obtain $P_{i}(\infty), \quad i=1,2,3, \cdots, 17$.

States $0,1,2,3,4,5,6$ and 7 in the states of the system IV above are operational states and states 1, 2, 3, $\cdots$, 17 are busy period states, putting (21) in the last rows of (20), the system availability, busy period and profit function are given by:

$$
\begin{gathered}
A_{T 4}(\infty)=P_{0}(\infty)+P_{1}(\infty)+P_{2}(\infty)+P_{3}(\infty)+P_{4}(\infty)+P_{5}(\infty)+P_{6}(\infty)+P_{7}(\infty)=\frac{H_{7}}{D_{4}} \\
B_{T 4}(\infty)=P_{1}(\infty)+P_{2}(\infty)+P_{3}(\infty)+P_{4}(\infty)+P_{5}(\infty)+P_{6}(\infty)+\cdots+P_{17}(\infty)=\frac{H_{8}}{D_{4}}
\end{gathered}
$$

The expected profit $\mathrm{PF}_{4}$ per unit time incurred to the system in the steady-state is given by:

Profit $=$ total revenue generated - accumulated cost incurred due corrective maintenance to the failed system/ subsystems/units.

Thus

$$
P F_{4}=C_{0} A_{T 4}(\infty)-C_{1} B_{T 4}(\infty)
$$




\section{Numerical Illustration}

In this section, we numerically obtained and compared the results for system availability and profit function for the developed models. The objectives here are to analyze graphically the effects of system parameters on availability and profit and make comparison for different values of $n$. For each model the following set of parameters values are fixed throughout the simulations for consistency.

$$
\begin{gathered}
\beta_{1}=0.4, \quad \beta_{2}=0.3, \beta_{11}=0.3, \beta_{12}=0.1, \beta_{13}=0.2, \quad \beta_{14}=0.3, \\
\alpha_{1}=0.2, \quad \alpha_{11}=0.3, \alpha_{2}=0.3, \alpha_{12}=0.4, \alpha_{13}=0.4, \alpha_{14}=0.5, \\
C_{0}=100,000, C_{1}=20,000 .
\end{gathered}
$$

It is can be seen from Figure 1, that availability increases with increase in repair rate $\alpha_{1}$ for $n=2,3,4,5$ and also the availability increases as $n$ increases. It is evident from Figure 1 that as $n$ increases, the steady state availability also increases. The result in Figure 1 also shows that steady state availability increases with increase in repair and provision of more standby units. Figure 2 shows that the availability decreases with increase in failure rate $\beta_{1}$. However, availability for $n=2, n=3, n=4$ decreases more compared to when $n=5$. Here the optimal availability result with respect to $\beta_{1}$ is when $n=5$. The result here indicates that the availability of the system with more standby units tend to decrease slightly than the system with less standby units. Figure 3 shows that the generated profit increases with increase in repair rate $\alpha_{1}$ for $n=2,3,4,5$. The profit is higher when $n=5$ than when $n=2,3$, 4. It is evident here that provision of more standby units lead to increase in the generated profit. Figure 4 shows that the generated profit decreases with increase in failure rate $\beta_{1}$. However, the generated profit for $n=2, n=3, n=4$ decreases more compared to when $n=5$. Here the optimal profit with respect to $\beta_{1}$ is when $n=5$. This indicates that the generated profit of the system with more standby units tend to decrease slightly than the system with less standby units. These numerical results are summarized in Table 1.

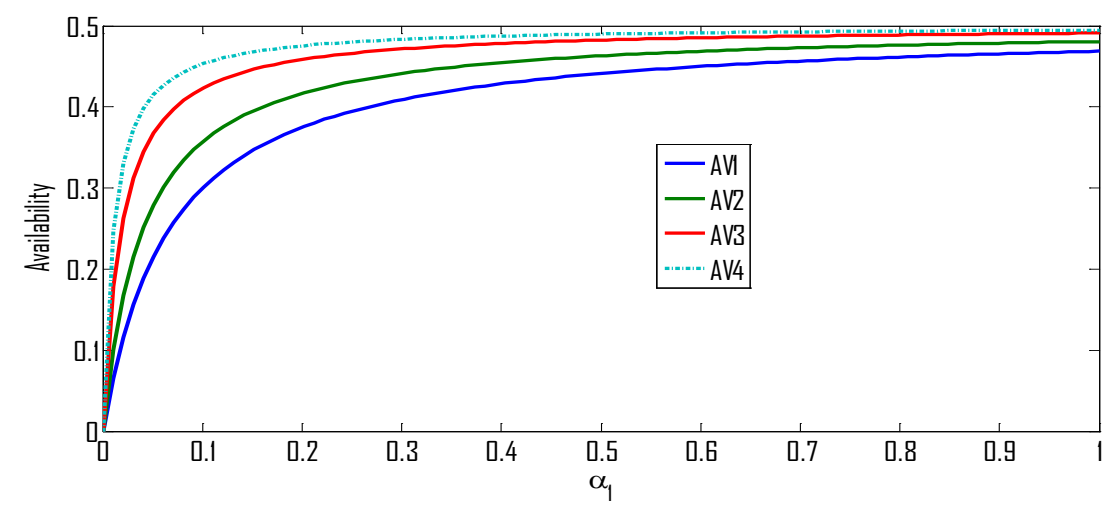

Figure 1. Availability against $\alpha_{1}$.

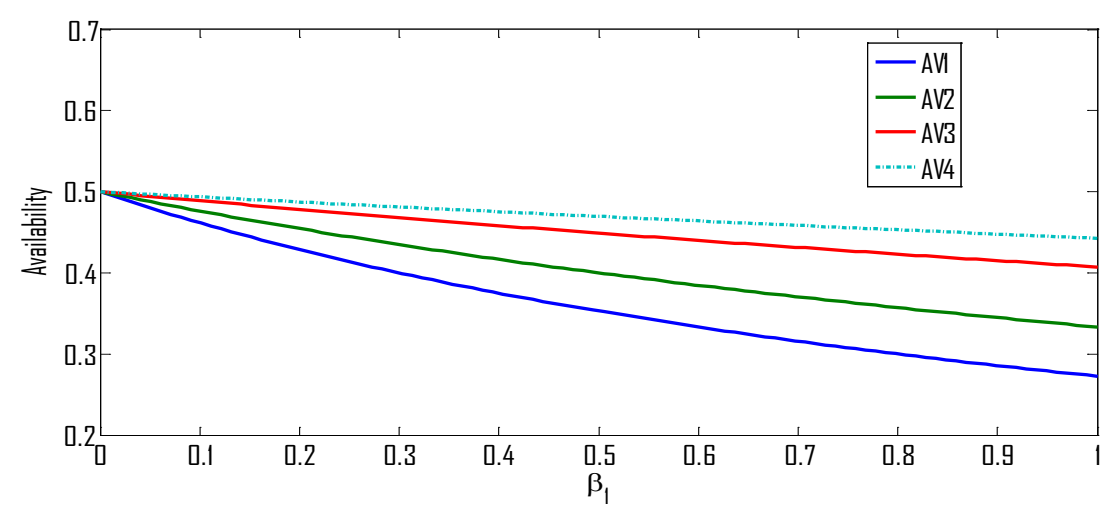

Figure 2. Availability against $\beta_{1}$. 


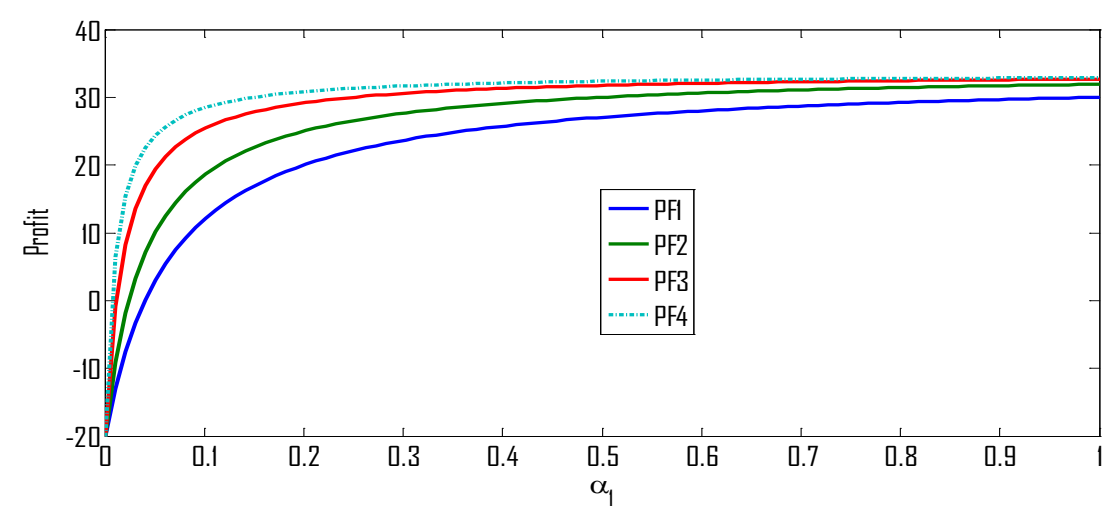

Figure 3. Profit against $\alpha_{1}$.

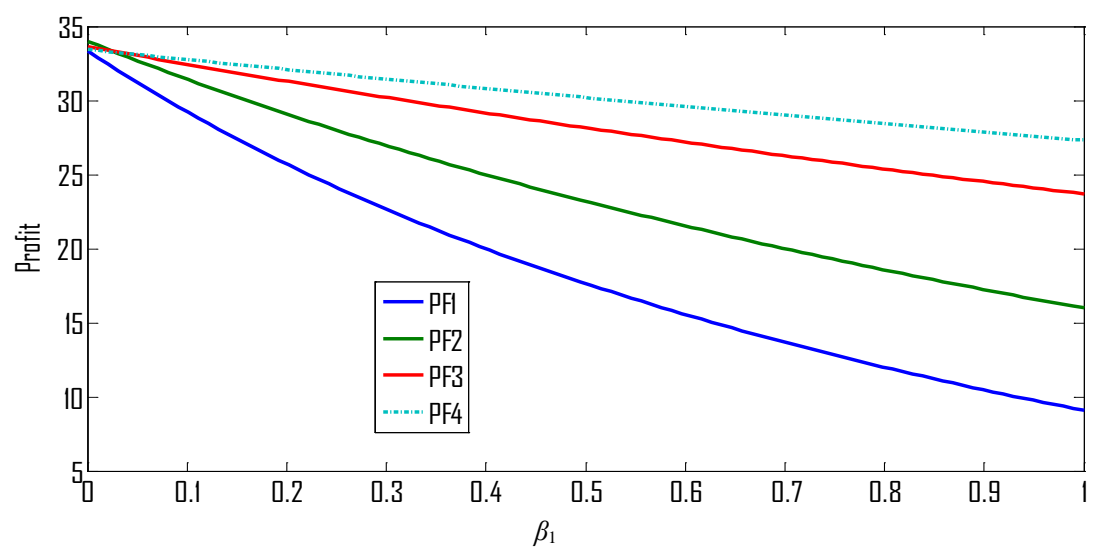

Figure 4. Profit against $\beta_{1}$.

Table 1. Comparison of availability and profit for $n=2,3,4,5$.

\begin{tabular}{ccc}
\hline Parameter & Range of parameter & Results \\
\hline$\beta_{1}$ & $0 \leq \beta_{1} \leq 1$ & $A_{r 4}>A_{r 3}(\infty)>A_{T 2}(\infty)>A_{r 1}$ \\
& & $P F_{4}>P F_{3}(\infty)>P F_{2}(\infty)>P F_{1}$ \\
$\alpha_{1}$ & $0 \leq \alpha_{1} \leq 1$ & $A_{r 4}>A_{r 3}(\infty)>A_{r 2}(\infty)>A_{r 1}$ \\
& $P F_{4}>P F_{3}(\infty)>P F_{2}(\infty)>P F_{1}$ \\
\hline
\end{tabular}

\section{Conclusion}

In this paper, we constructed four different series-parallel systems consisting of subsystems $A, B$ and $C$. Subsystems $A$ and $B$ are cold standby with subsystem $A$ containing linear consecutive $k$-out-of- $n$ units while subsystem $B$ and $C$ consist of a single unit each. We developed the explicit expressions for the availability, busy period and profit for the four systems and performed a comparative analysis. It is interesting to see that as the number of units in subsystem $A$ increases, the availability and profit also increase. Parametric investigation of various system parameters on system availability and profit function has been captured. It is evident from Table 1 that the system with $n=5$ units in subsystem $A$ is optimal. The results of this paper are found to be highly beneficial to maintenance managers, reliability engineers, plant management and system designers for the proper maintenance analysis, decision making, system safety, and performance evaluation.

\section{References}

[1] Hu, l., Yue, D. and Li, J. (2012) Availability Analysis and Design Optimization for a Repairable Series-Parallel System 
with Dependencies, International Journal of Innovative Computing, Information and Control, 8, 6693-6705.

[2] Khatab, A., Nahas, N. and Nourelfath, M. (2009) Availbilty of K-Out-of-N: G Systems with Non-Identical Components Subject to Repair Priorities. Reliability Engineering \& System Safety, 94, 142-151. http://dx.doi.org/10.1016/j.ress.2008.02.017

[3] Krishnan, R. and Somasundaram, S. (2012) Reliability and Profit Analysis of Repairable K-Out-of-N System with Sensor. European Journal of Scientific Research, 67, 215-222.

[4] Juang, Y.-S., Lin, S.-S. and Kao, H.-P. (2008) A Knowledge Management System for Series-Parallel Availability Optimization and Design. Expert Systems with Applications, 34, 181-193. http://dx.doi.org/10.1016/j.eswa.2006.08.023

[5] Levitin, G. (2002) Optimal Series-Parallel Topology of Multi-State System with Two Failure Modes. Reliability Engineering \& System Safety, 77, 93-107. http://dx.doi.org/10.1016/S0951-8320(02)00034-0

[6] Li, C.Y., Chen, X., Yi, X.S. and Tao, J.Y. (2010) Heterogeneous Redundancy Optimization for Multi-State SeriesParallel Systems Subject to Common Cause Failures. Reliability Engineering and System Safety, 95, 202-207. http://dx.doi.org/10.1016/j.ress.2009.09.011

[7] Wang, S. and Wadata, J. (2009) Reliability Optimization of a Series-Parallel System with Fuzzy Random Lifetimes. International Journal of Innovative Computing, Information and Control, 5, 1547-1558.

[8] Wang, K.H. and Chen, Y.J. (2009) Comparative Analysis of Availability between Three Systems with General Repair Times, Reboot Delay and Switching Failures. Applied Mathematics and Computation, 215, 384-394. http://dx.doi.org/10.1016/j.amc.2009.05.023

[9] Wang, K.-H., Yen, T.-C. and Fang, Y.-C. (2012) Comparison of Availability between Two Systems with Warm Standby Units and Different Imperfect Coverage. Quality Technology and Quantitative Management, 9, $265-282$. 


\section{Appendix}

\section{Notations}

$A_{j s}, A_{j O}, A_{j R}, A_{j W}, A_{j G}$ : Unit in subsystem $\mathrm{A}$ is in standby, in operation, failed and under repair, failed and waiting for repair, idle for $j=1,2,3,4,5$

$B_{S}, B_{O}, B_{R}, B_{G}$ : Subsystem $B$ is in standby, operation, failed and is under repair, is idle

$C_{O}, C_{R}, C_{G}$ : Subsystem $C$ is in operation, failed and is under repair, is idle

$T$ : Time to failure of the system

$A_{T i}, \quad B_{T i}, \quad P F_{i}$ : Steady-state availability, Busy period and Profit function for $i=1,2,3,4$

$P_{m}(t)$ : Probability that the system is in state $S_{m}$ at $t \geq 0$ for $m=0,1,2,3, \cdots, 17$

$\beta_{1 w}, \alpha_{1 w}$ : Failure and repair rate of unit $A_{1 w}$ in subsystem A for $w=1,2,3,4$

$\beta_{1}, \alpha_{1}$ : Failure and repair rate of subsystem $B$

$\beta_{2}, \alpha_{2}$ : Failure and repair rates of subsystem $C$

$n$ : Total number of units in subsystem $A$

$C_{0}$ : Revenue generated when the system is in working state and no income when in failed state

$C_{1}$ : Cost of each repair for failed system/subsystems/units

$D_{1}=\alpha_{2} \beta_{1} \beta_{11} \beta_{12}+\alpha_{1} \alpha_{2} \beta_{11} \beta_{12}+\alpha_{2} \beta_{1} \beta_{2} \beta_{11}+\alpha_{1} \alpha_{2} \alpha_{12} \beta_{11}+\alpha_{1} \alpha_{2} \beta_{2} \beta_{11}+\alpha_{1} \alpha_{2} \alpha_{11} \alpha_{12}+\alpha_{1} \beta_{2} \beta_{11} \beta_{12}+\alpha_{1} \alpha_{12} \beta_{2} \beta_{11}$

$+\alpha_{1} \beta_{2}^{2} \beta_{11}+\alpha_{1} \alpha_{11} \alpha_{12} \beta_{2}$,

$D_{2}=\alpha_{1} \alpha_{2} \alpha_{12} \beta_{11}+\alpha_{1} \alpha_{2} \beta_{11} \beta_{12}+\alpha_{1} \alpha_{2} \alpha_{11} \alpha_{12}+\alpha_{1} \alpha_{2} \alpha_{11} \beta_{12}+\alpha_{1} \alpha_{12} \beta_{2} \beta_{11}+\alpha_{1} \beta_{2} \beta_{11} \beta_{12}+\alpha_{1} \alpha_{11} \alpha_{12} \beta_{2}+\alpha_{1} \alpha_{11} \beta_{2} \beta_{12}$

$+\alpha_{2} \beta_{1} \beta_{11} \beta_{12}+\alpha_{1} \alpha_{11} \beta_{1} \beta_{12}$,

$D_{3}=\alpha_{1} \alpha_{2} \alpha_{11} \alpha_{13} \beta_{12}+\alpha_{1} \alpha_{2} \alpha_{11} \beta_{12} \beta_{13}+\alpha_{1} \alpha_{2} \alpha_{11} \alpha_{12} \alpha_{13}+\alpha_{1} \alpha_{2} \alpha_{13} \beta_{11} \beta_{12}+\alpha_{1} \alpha_{2} \alpha_{12} \alpha_{13} \beta_{11}+\alpha_{1} \alpha_{2} \beta_{11} \beta_{12} \beta_{13}$

$+\alpha_{1} \alpha_{11} \alpha_{13} \beta_{2} \beta_{12}+\alpha_{1} \alpha_{11} \beta_{2} \beta_{12} \beta_{13}+\alpha_{1} \alpha_{11} \alpha_{12} \alpha_{13} \beta_{2}+\alpha_{1} \alpha_{13} \beta_{2} \beta_{11} \beta_{12}+\alpha_{1} \alpha_{12} \alpha_{13} \beta_{2} \beta_{11}+\alpha_{1} \beta_{2} \beta_{11} \beta_{12} \beta_{13}$

$+\alpha_{2} \alpha_{11} \beta_{1} \beta_{12} \beta_{13}+\alpha_{2} \beta_{1} \beta_{11} \beta_{12} \beta_{13}$,

$D_{4}=\alpha_{1} \alpha_{2} \beta_{11} \beta_{12} \beta_{13} \beta_{14}+\alpha_{1} \alpha_{2} \beta_{11} \beta_{12} \beta_{13} \beta_{14}+\alpha_{1} \alpha_{2} \alpha_{13} \alpha_{14} \beta_{11} \beta_{12}+\alpha_{1} \alpha_{2} \alpha_{12} \alpha_{13} \alpha_{14} \beta_{11}+\alpha_{1} \alpha_{2} \alpha_{11} \beta_{12} \beta_{13} \beta_{14}$

$+\alpha_{1} \alpha_{2} \alpha_{11} \alpha_{14} \beta_{12} \beta_{13}+\alpha_{1} \alpha_{2} \alpha_{11} \alpha_{13} \alpha_{14} \beta_{12}+\alpha_{1} \alpha_{2} \alpha_{11} \alpha_{12} \alpha_{13} \alpha_{14}+\alpha \beta_{2} \beta_{11} \beta_{12} \beta_{13} \beta_{14}+\alpha_{1} \alpha_{14} \beta_{2} \beta_{11} \beta_{12} \beta_{13}$

$+\alpha_{1} \alpha_{13} \alpha_{14} \beta_{2} \beta_{11} \beta_{12}+\alpha_{1} \alpha_{12} \alpha_{13} \alpha_{14} \beta_{2} \beta_{11}+\alpha_{1} \alpha_{11} \beta_{2} \beta_{12} \beta_{13} \beta_{14}+\alpha_{1} \alpha_{11} \alpha_{14} \beta_{2} \beta_{12} \beta_{13}+\alpha_{1} \alpha_{11} \alpha_{13} \alpha_{14} \beta_{2} \beta_{12}$

$+\alpha_{1} \alpha_{11} \alpha_{12} \alpha_{13} \alpha_{14} \beta_{2}+\alpha_{2} \beta_{1} \beta_{11} \beta_{12} \beta_{13} \beta_{14}+\alpha_{2} \alpha_{11} \beta_{1} \beta_{12} \beta_{13} \beta_{14}$,

$H_{1}=\alpha_{1} \alpha_{2} \alpha_{11} \alpha_{12}+\alpha_{1} \alpha_{2} \alpha_{12} \beta_{11}+\alpha_{1} \alpha_{2} \beta_{11}\left(\beta_{12}+\beta_{2}\right)$

$H_{2}=\alpha_{1} \alpha_{2} \alpha_{12} \beta_{11}+\alpha_{1} \alpha_{2} \beta_{11}\left(\beta_{2}+\beta_{12}\right)+\alpha_{1} \alpha_{11} \alpha_{12} \beta_{2}+\alpha_{2} \beta_{1} \beta_{11}\left(\beta_{2}+\beta_{12}\right)+\alpha_{1} \beta_{2} \beta_{11}\left(\alpha_{12}+\beta_{12}+\beta_{2}\right)$

$H_{3}=\alpha_{1} \alpha_{2} \alpha_{11} \alpha_{12}+\alpha_{1} \alpha_{2} \alpha_{12} \beta_{11}+\alpha_{1} \alpha_{2} \alpha_{11} \beta_{12}+\alpha_{1} \alpha_{2} \beta_{11} \beta_{12}$

$H_{4}=\alpha_{1} \alpha_{2} \alpha_{12} \beta_{11}+\alpha_{1} \alpha_{2} \alpha_{11} \beta_{12}+\alpha_{1} \alpha_{2} \beta_{11} \beta_{12}+\alpha_{1} \alpha_{11} \alpha_{12} \beta_{2}+\alpha_{1} \alpha_{12} \beta_{2} \beta_{11}+\alpha_{2} \alpha_{11} \beta_{1} \beta_{12}+\alpha_{1} \alpha_{11} \beta_{2} \beta_{12}+\alpha_{2} \alpha_{1} \beta_{11} \beta_{12}$

$+\alpha_{1} \alpha_{2} \beta_{11} \beta_{12}$

$H_{5}=\alpha_{1} \alpha_{2} \alpha_{11} \alpha_{12} \alpha_{13}+\alpha_{1} \alpha_{2} \alpha_{12} \alpha_{13} \beta_{11}+\alpha_{1} \alpha_{2} \alpha_{11} \alpha_{13} \beta_{12}+\alpha_{1} \alpha_{2} \alpha_{13} \beta_{11} \beta_{12}+\alpha_{1} \alpha_{2} \beta_{11} \beta_{12} \beta_{13}+\alpha_{1} \alpha_{2} \alpha_{11} \beta_{12} \beta_{13}$

$H_{6}=\alpha_{1} \alpha_{2} \alpha_{12} \alpha_{13} \beta_{11}+\alpha_{1} \alpha_{2} \alpha_{11} \alpha_{13} \beta_{12}+\alpha_{1} \alpha_{2} \alpha_{13} \beta_{11} \beta_{12}+\alpha_{1} \alpha_{2} \beta_{11} \beta_{12} \beta_{13}+\alpha_{1} \alpha_{2} \alpha_{11} \beta_{12} \beta_{13}+\alpha_{1} \alpha_{12} \alpha_{13} \beta_{2} \beta_{11}$

$+\alpha_{1} \alpha_{13} \beta_{2} \beta_{11} \beta_{12}+\alpha_{2} \beta_{1} \beta_{11} \beta_{12} \beta_{13}+\alpha_{1} \beta_{2} \beta_{11} \beta_{12} \beta_{13}+\alpha_{2} \alpha_{11} \beta_{1} \beta_{12} \beta_{13}+\alpha_{1} \alpha_{11} \beta_{2} \beta_{12} \beta_{13}+\alpha_{1} \alpha_{11} \alpha_{12} \alpha_{13} \beta_{2}$

$+\alpha_{1} \alpha_{2} \alpha_{11} \beta_{2} \beta_{12}$,

$H_{7}=\alpha_{1} \alpha_{2} \alpha_{11} \alpha_{12} \alpha_{13} \alpha_{14}+\alpha_{1} \alpha_{2} \alpha_{12} \alpha_{13} \alpha_{14} \beta_{11}+\alpha_{1} \alpha_{2} \alpha_{11} \alpha_{13} \alpha_{14} \beta_{12}+\alpha_{1} \alpha_{2} \alpha_{13} \alpha_{14} \beta_{11} \beta_{12}+\alpha_{1} \alpha_{2} \alpha_{14} \beta_{11} \beta_{12} \beta_{13}$

$+\alpha_{1} \alpha_{2} \beta_{11} \beta_{12} \beta_{13} \beta_{14}+\alpha_{1} \alpha_{2} \alpha_{11} \alpha_{14} \beta_{12} \beta_{13}+\alpha_{1} \alpha_{2} \alpha_{11} \beta_{12} \beta_{13} \beta_{14}$,

$H_{8}=\alpha_{1} \alpha_{2} \alpha_{12} \alpha_{13} \alpha_{14} \beta_{11}+\alpha_{1} \alpha_{2} \alpha_{11} \alpha_{13} \alpha_{14} \beta_{12}+\alpha_{1} \alpha_{2} \alpha_{13} \alpha_{14} \beta_{11} \beta_{12}+\alpha_{1} \alpha_{2} \alpha_{14} \beta_{11} \beta_{12} \beta_{13}+\alpha_{1} \alpha_{2} \beta_{11} \beta_{12} \beta_{13} \beta_{14}$

$+\alpha_{1} \alpha_{2} \alpha_{11} \alpha_{14} \beta_{12} \beta_{13}+\alpha_{1} \alpha_{2} \alpha_{11} \beta_{12} \beta_{13} \beta_{14}+\alpha_{1} \alpha_{11} \alpha_{12} \alpha_{13} \alpha_{14} \beta_{2}+\alpha_{1} \alpha_{12} \alpha_{13} \alpha_{14} \beta_{2} \beta_{11}+\alpha_{1} \alpha_{13} \alpha_{14} \beta_{2} \beta_{11} \beta_{12}$

$+\alpha_{1} \alpha_{14} \beta_{2} \beta_{11} \beta_{12} \beta_{13}+\alpha_{2} \beta_{1} \beta_{11} \beta_{12} \beta_{13} \beta_{14}+\alpha_{1} \beta_{2} \beta_{11} \beta_{12} \beta_{13} \beta_{14}+\alpha_{1} \alpha_{11} \alpha_{13} \alpha_{14} \beta_{2} \beta_{12}+\alpha_{1} \alpha_{11} \alpha_{14} \beta_{2} \beta_{12} \beta_{13}$

$+\alpha_{2} \alpha_{11} \beta_{1} \beta_{12} \beta_{13} \beta_{14}+\alpha_{1} \alpha_{11} \beta_{2} \beta_{12} \beta_{13} \beta_{14}$. 
Scientific Research Publishing (SCIRP) is one of the largest Open Access journal publishers. It is currently publishing more than 200 open access, online, peer-reviewed journals covering a wide range of academic disciplines. SCIRP serves the worldwide academic communities and contributes to the progress and application of science with its publication.

Other selected journals from SCIRP are listed as below. Submit your manuscript to us via either submit@scirp.org or Online Submission Portal.
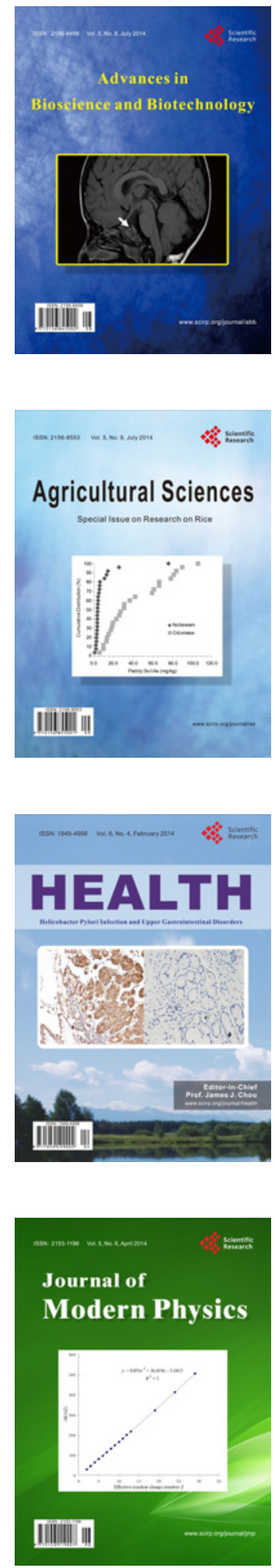
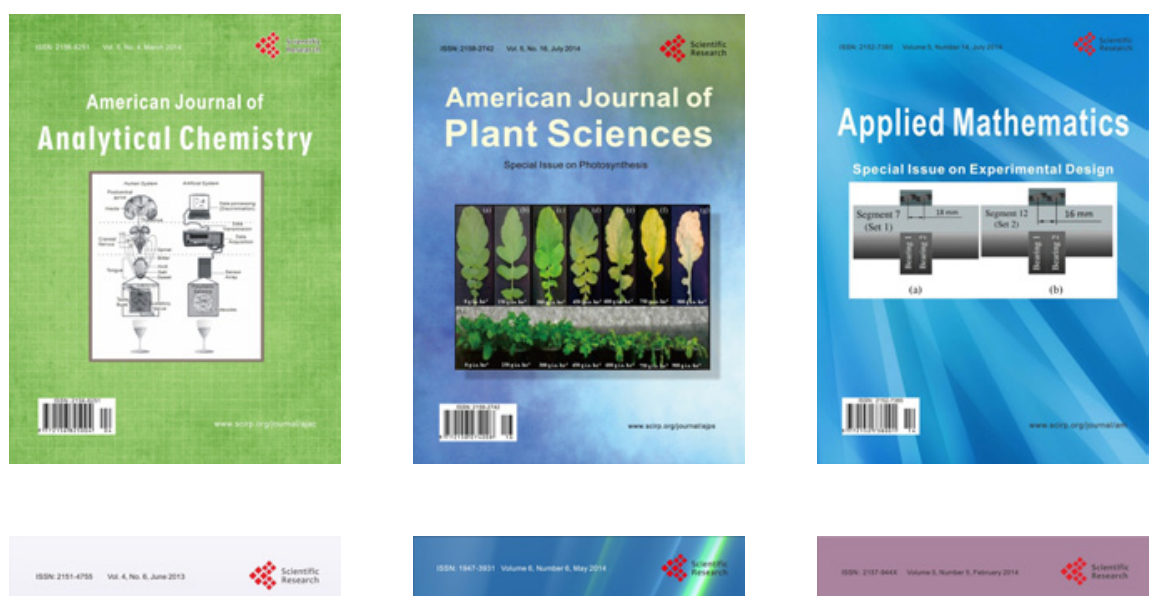

Creative Education
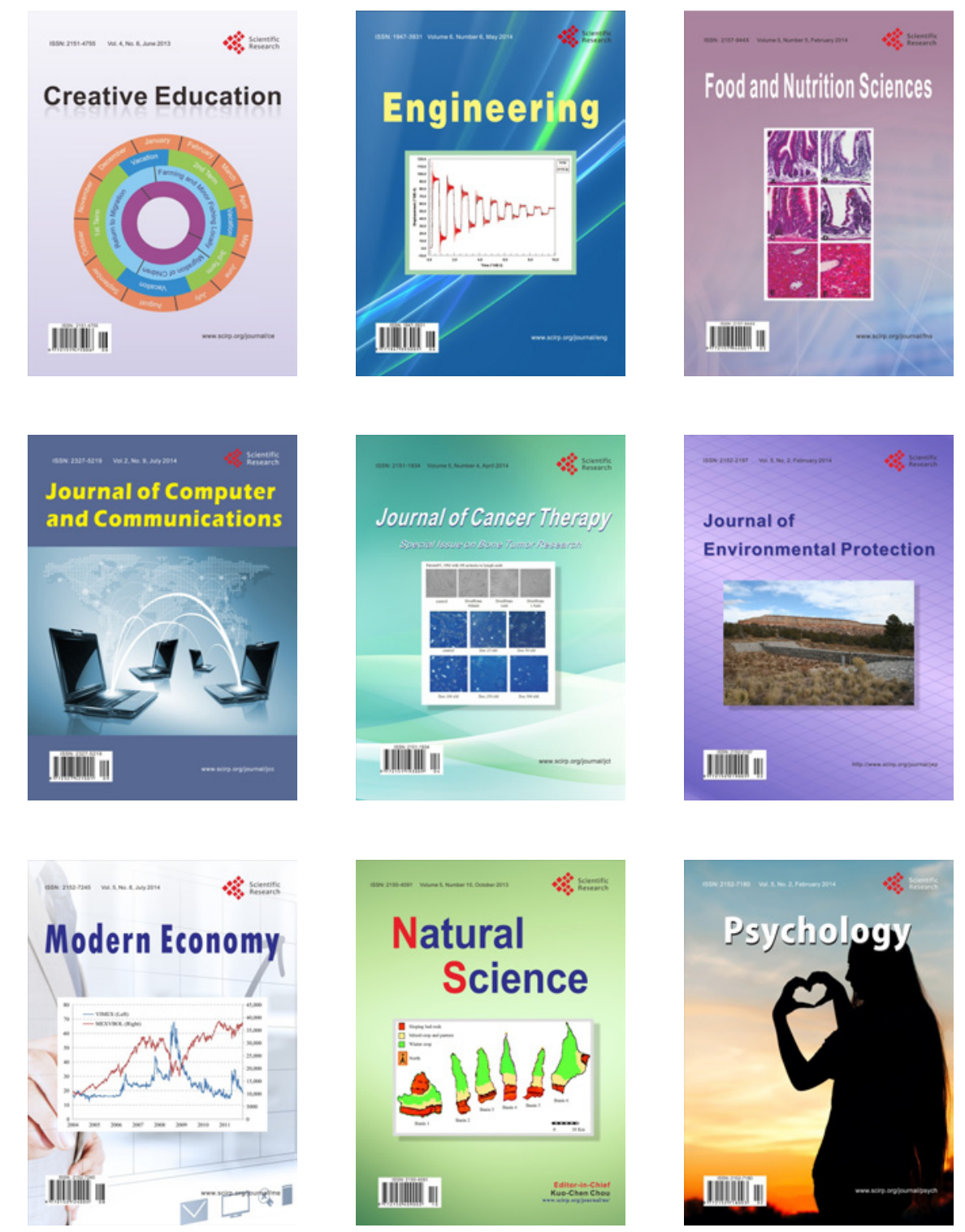